\title{
On the Current Situation and Development Tendency of Smart Home in China
}

\author{
Tianle Wen
}

Century College, Beijing University of Posts and Telecommunications, Yanqing, 102101, China

\author{
Keywords: Internet of Things, Smart, Home
}

\begin{abstract}
Based on the research and analysis of the current situation of the development of the Internet of things at home and abroad, this paper position the application object of Internet of things for the smart home. In accordance with the possible technique difficulties of the smart home, it puts forward the solutions of the smart home environment based on the Internet of things. The scheme contrasts the different schemes of wireless access and selects the wireless sensor network based on ZigBee. Combined with the mature internet and mobile cellular network, the framework of home environment system is established. The S3C6410 hardware platform based on ARM is selected in the gateway hardware options. The development environment based on Android is selected in the handset options. The process of the overall design realizes the physical information transition to make the signaling and data of the smart home system flow smoothly among the three networks.
\end{abstract}

\section{Basic Concept and Key Technologies of IoT}

Origin and Definition of IoT. The concept of the Internet of things does not appear out of void. It comes from the integration of scholars' achievements in many fields. KevinAshton, the author of the word, put forward the concept in the process of the research of supply chain management.

The concept of the Internet of things was firstly proposed in 1999. The internet of things is no longer the traditional sense of the internet, which is based on the internet as the core. It is the expansion of the internet. It is also the terminal from the traditional personal computers, mobile phones and other devices, extended to a variety of information exchange objects. Therefore, we can define the Internet through a variety of wireless communication systems, in accordance with the contract agreement, any objects linked to the Internet, so as to complete the transfer of information, further implementation of new network analysis to the information of the object. The wireless communication system includes radio frequency technology, laser scanning technology, infrared technology, global positioning system and so on.

Although a large number of new technologies are used in the internet of things, these new technologies are based on the original internet. So, if there is no internet as a foundation, the internet of things cannot work.

Internet of things is different from the internet. It is the development of the internet. In essence, it is an extension of the internet, but it is definitely not in the traditional sense of the internet. Interpersonal communication can be achieved through the internet, and the information transfer of things need the internet of things. In this network, the system can identify, locate, track, monitor and trigger the corresponding events automatically and in real time.

The object must be collected related information to understand an object through the internet. We upload the information to the Internet for people to browse, which leads to a lot of work to do. It is difficult to understand the dynamic changes. It is the object itself in the expression, through the implantation of the sensor chip in the object, the use of wireless communication networks, and the Internet connection, the realization of information transmission. In this way, people can not only communicate with objects, but also communicate between objects and objects. Therefore, the network is realized in the virtual network world, which is the physical realization of the Internet of things and the real-world network.

System Structure. The internet of things can be divided into three layers: perception layer, network layer and application layer. The perception layer is located at the bottom of the Internet of 
things architecture and is responsible for the collection of relevant data. It mainly includes the wired/wireless sensor networks, various types of sensor equipment, RFID and video capture equipment. The sensing layer basic technology mainly includes the sensor and the controller technology, as well as the short distance transmission technology. The access capability of the sensing layer plays a fundamental role in the realization of the Internet of things. The network layer is based on the communication network and the Internet on the current set up, the key technology including the existing communication technologies including terminal technology and provide communication capability for all kinds of terminal communication module, the network layer can not only use the user whenever and wherever possible to receive the service. The combination with network technology through the collaborative work of wired and wireless is more important, providing intelligent access network selection mode for the user.

\section{Application Layer Design of Smart Home}

Development of Service Function of Web. Web service is a remote call convention, which can shield the hardware architecture, operating systems and programming languages and other factors. Based on the Internet of things, smart home not only achieves home furnishing management and operation inside the home appliance, but also should make the internal home furnishing operation according to the open mind of Internet of things. It can be implemented through standard network call. But this operation is aimed at a particular system and a specific device. If it is not an Android operating system phone, which is iOS or Windows Phone, the program needs to be redeveloped.

One way to solve this problem is Web service. The Web service is based on the standard network call convention, the operating system, or the programming language, and the remote execution of the program through the same call command and the return of the result. In addition to the general function of the message publishing and information storage, the server is the most important role as a Web service publishing platform. As mentioned earlier, there are three entities involved in the whole process of the design of a service-oriented architecture. They are the service provider, the publisher of the service and the consumer of the service. The user of the remote call is the consumer, the home gateway is the service provider, and the gateway server is the center of service publication and registration. As a complete implementation of the Web service, a publisher will not only be able to implement the service, but also explicitly specify the WSDL document for the service.

All the realization processes of Web service are: first, program to complete the function, for the use of language programming. Second, according to the programming function module, the WSDL document is generated. This WSDL document specifies the name, description, calling parameter and return value of the Web service and the location and port of the call. The differences of different programming languages are unified. Third, upload the service module to the server. Upload the WSDL file to the UDDI registration center, and register it as a new service. Now, a Web service can be discovered and called by the client.

All the realization processes of Web invocation are: Above all, if it is the first invocaation, we can go to the UDDI registry to find their own required services. Second, UDDI registry can help us search for this service and provide the corresponding service WSDL file. The client parses the file. Third, according to the analytical results, the system the automatic generates the code to invoke this service. Code will be based on the different platforms and the use of different programming languages. The invocation will be done later. The development kit of eclipse+tomcat+axis2 is used.

Overall Design Thinking of Intelligent Agent. Based on the intelligent home system, the intelligent agent mainly includes three kinds of agents: management agent, environment agent and execution agent. There is also a user agent specifically designed to interact with users. Each agent represents a type of device or individual. The management agent is in the central position. It is responsible for coordinating the work of all agents and issuing control orders.

As a platform interface user and the user agent, the deployment location of the agents is not fixed according to the user environment and the transfer of equipment. The management agent, the execution agent and the environment agent are all located in the home environment. The role of the 
environmental agent is to collect all the sensor information, and the information preprocessing. Its service object is a variety of temperature and humidity sensors, door and window magnetic stripe and harmful gas monitoring information. The information collected will be used as a basis for making decisions on the home agent. As the interface of all the actuators in the home, the implementation of the agent is responsible for the unified operation of all household equipment. The management agent acts as the manager of all agents to coordinate the information flow of the agent platform. It is also a decision maker in the agent platform. On the one hand, it will be based on real-time environmental information and information stored in the database to notify the executive agent to complete the work. On the one hand, it can also receive commands directly from the user.

Detailed Design of Intelligent Agent. Multi agent platform is a virtual platform. It is run by the JADE to build a cross platform and cross operating system environment. Different agents can be physically located in different environments. This system, implementation of the agent and the environment agency must be located in the smart home environment. The user agents generally follow the user in the mobile terminal, and management agent position is relatively flexible. It can be located in the smart home environment and uniformly managed in the area on the server.

Task division. There are four kinds of agents in the system. The most important is management agent, which is the first agent in the system, and is responsible for other agents in the management system. Management agents involved in the tasks include the following aspects: first, start the management agents only after the agent platform is set. After the initialization of the management agent, the environment agent, the execution agent and the user agent are started. In this process, the management agent should also register, prepare the database and initialize the user interface. Second, when all agents work properly, the management agent enters the home environment monitoring cycle. The management agent periodically sends a command to the environment agent to obtain environmental information, and automatically analyzes the environmental information and triggers the default behavior according to the built-in rules in the database. Third, management agent will listen to other active foreign news, such as the user agent made the request, agent management interface and other messages. The management agent generates an internal command by analyzing the intent of these messages to operate the home's sensors and actuators.

Agent platform ontology design. The concept of ontology is used in the JADE proxy platform, but in this case, the more popular one is the lexicon. In the face of the characteristics of the smart home, the home of some of the terms and expressions, together constitute the smart home ontology. The role of the design ontology is to ensure that the interpretation of the same terms between the agents is the same, not to the misunderstanding of the concept. In only a Home Furnishing environment application, of course will not have what meaning, but when an agent platform is not only a Home Furnishing management environment, but also the management of other aspects of life, then it is possible to produce a sense of misunderstanding. Therefore, it is necessary to establish the ontology based on the home, to determine the vocabulary and operation name. This is the reasons of the introduction of the concept of ontology of JADE platform.

According to the design of application scenarios, we need to consider all aspects of the problem. The more careful consideration, the more perfect the ontology construction is. It is possible to make the interaction between agents more clear, more efficient implementation of the agent, the effect is more obvious. Consider the relationship between the various concepts of the family space and the relationship between the various relationships, which will directly affect the intelligence of the intelligent home.

Communication between agents. The above is the ontology used in the platform. The agents interact with the various words in the ontology. The inter agent interaction model is based on the JADE platform provided by the ACL (Agent Communication Language) mechanism. Each message in the ACL contains the following elements: First, Sender and Receivers. Sender and receiver are specified by the platform only AID. The second is the communicative intention/primitives. It is provided by the JADE platform several communication primitives, covering a variety of communication purposes. The commonly used language is REQUEST, which is an agent requires other agents to perform a behavior; INFORM: proxy notification to other agents of an assertion; 
REFUSE: agent to reject a behavior and so on. These agents express the purpose of inter-agency communication. The third are content, language and ontology. Content specifies the content of the message. Language specifies the encoding used by the message. Ontology specifies the vocabulary in the message which comes from the vocabulary, so that the receiver and sender do not make the same word ambiguous.

Different agents use ACLMessage to communicate with each other and analyze the Content. Combined with the intention of communication, it can be informed that these messages are need to be queried, performed, or to be determined true or false. Then, through a simple if-then judgment, we can get the next task to be executed. Management agents will give these tasks to the appropriate agents to perform.

\section{References}

[1] Aiello M, Dustdar S. Are our homes ready for services? a domotic infrastructure based on the web service stack [J]. Pervasive and Mobile Computing, 2008, 4(4): 506-525.

[2] He Yanyan, Research on the development and tendency of smart home based on Internet of things [J]. Wireless Internet Technology, 2016(22): 15-16.

[3] Tan Yuanyuan, Geng Daoshuang, Household Smart Product Design under the Lifestyle [J]. Packaging Engineering, 2016, 37(22): 108-113.

[4] Guan Qilin, Current Situation and Problems Analysis on Smart Home [J]. City \& House, 2016, 23(3): 40-42.

[5] Ha E T, Kim S, Kim J, et al. Learning a Bayesian Network for Situation-Aware Smart Home Service: A Case Study with a Robot Vacuum Cleaner [J]. International Science Index Computer and Information Engineering, 2015, 2(2): 877-884. 